Table 2. Type of flare after IS discontinuation in 105 SLE remitted patients.

\begin{tabular}{lcc}
\hline Type of flare & Number $(\%)$ & Severe flare, number (\% of all flares) \\
\hline Arthritis & $5(19.2)$ & $2(7.7)$ \\
Skin manifestations & $6(23.1)$ & $1(3.9)$ \\
Serosal involvement & $2(7.7)$ & 0 \\
Haematological involvement & $5(19.2)$ & $2(7.7)$ \\
Lupus nephritis & $8(30.8)$ & $8(30.8)$ \\
Total & 26 & $13(50)$ \\
\hline
\end{tabular}

Conclusion: In our SLE cohort, the withdrawal of IS therapy in remitted patients did not seem to influence damage progression in the medium-term.

Disclosure of Interests: : None declared

DOI: 10.1136/annrheumdis-2019-eular.7808

\section{OP0248 THE IMPACT OF EXERCISE ON HAND FUNCTION AND QUALITY OF LIFE OF PATIENTS WITH SYSTEMIC LUPUS ERYTHEMATOSUS: A RANDOMIZED CONTROLLED TRIAL}

Kyriaki Keramiotou ${ }^{1,2}$, Christos Anagnostou ${ }^{2}$, Antonis Galanos ${ }^{3}$, Evaggelia Kataxaki ${ }^{2}$, Petros Sfikakis ${ }^{4}$, Maria Tektonidou ${ }^{5}{ }^{1}$ National and Kapodistrian University of Athens, Medical School, Athens, Greece: ${ }^{2}$ Thriasio Hospital, Elefsina, Greece; ${ }^{3}$ National and Kapodistrian University of Athens, Athens, Greece, ${ }^{1}$ National and Kapodistrian University of Athens, Medical School, Athens, Greece; ${ }^{1}$ National and Kapodistrian University of Athens, Medical School, Athens, Greece

Background: Systemic Lupus Erythematosus (SLE) has a significant impact on the ability to perform daily activities and patients' quality of life. To date, no studies have examined the effect of exercise on hand function and quality of life of SLE patients.

Objectives: To determine the effect of exercise on hand strength, dexterity and performance of daily activities, and the quality of life of SLE patients.

Methods: A randomized, 24-week follow-up trial was designed. A total of 240 consecutive SLE patients fulfilling the SLICC classification criteria were evaluated. Sixty two patients who met the inclusion criteria [age $>18$ years, upper limb arthralgias, DASH (Disabilities of the Arm, Shoulder, and Hand) score $>10$ and stable drug regimen for $\geq 3$ months], were randomly assigned to the exercise group $(\mathrm{n}=32)$ or the routine care (control) group $(\mathrm{n}=30)$.

Patients in the exercise group received by the hand therapist a 30 min daily program at home of strengthening and stretching upper limb exercises for 12 weeks. Performance of daily activities was evaluated with the DASH and HAQ questionnaires, the grip and pinch strength with the Jamar dynamometer and pinch gauge tools respectively, the dexterity with the Purdue pegboard test, and the quality of life with the LUPUSQoL questionnaire, at 0,6,12 (end of the exercise program) and 24 weeks for both groups. SLE activity and cumulative organ damage were evaluated with the SLEDAI and SLICC/ACR-DI, respectively.

Results: There were no statistically significant differences between two groups in age $(p=0.062)$, sex $(p=0.346)$, disease duration $(p=0.065)$, SLEDAI $(p=0.937)$, SLICC/ACR-DI ( $p=0.242)$, number of tender joints $(p=0.703)$, and use of corticosteroids $(p=0.640)$, hydroxycloroquine $(p=0.985)$ or immunosupressives $(p=0.806)$. Thirty patients $(93.75 \%)$ from the exercise group completed the 12 week exercise program and $28(87.5 \%)$ were re-evaluated at 24 weeks; 30 patients $(100 \%)$ of control group completed the 24-week study.

The percent changes of DASH and HAQ scores were significantly lower (improvement) in the exercise group compared to control group at all-time points. Moreover, all percent changes of grip strength, pinch strength hand dexterity and the LupusQol score were significantly higher in the exercise group compared to control group at all-time points (Table 1).

Table 1. Comparison between groups of percent changes from baseline to 6,12 and 24 weeks for all parameters.

\begin{tabular}{|c|c|c|c|c|}
\hline & $\begin{array}{c}\text { Evaluation time } \\
\text { (weeks) }\end{array}$ & $\begin{array}{l}\text { Exercise } \\
\text { Group }\end{array}$ & $\begin{array}{l}\text { Control } \\
\text { Group }\end{array}$ & $\begin{array}{c}\text { P. } \\
\text { value }\end{array}$ \\
\hline \multirow[t]{3}{*}{ DASH score } & 6 & $-33.7(28.4)$ & $-1.3(33.8)$ & $<0.001$ \\
\hline & 12 & $-43.4(31.5)$ & $-11.2(34.5)$ & $<0.001$ \\
\hline & 24 & $-51.9(47.8)$ & $-7.1(54.1)$ & $<0.001$ \\
\hline \multirow[t]{3}{*}{ HAQ score } & 6 & $-20(49.8)$ & $49.77(39.9)$ & 0.013 \\
\hline & 12 & $-55(70.4)$ & $-9.72(42.2)$ & $<0.001$ \\
\hline & 24 & $-69.8(56.3)$ & $-9.13(60.4)$ & $<0.001$ \\
\hline \multirow[t]{3}{*}{ Grip strength (DH) } & 6 & $9.9(32.8)$ & $2.39(35.5)$ & 0.044 \\
\hline & 12 & $20.9(43.4)$ & $4.10(30.2)$ & $<0.001$ \\
\hline & 24 & 23.1 (41) & $13.51(36.8)$ & 0.020 \\
\hline
\end{tabular}

\begin{tabular}{lccccc}
$\begin{array}{l}\text { Pinch strength } \\
\text { (JAW) }\end{array}$ & & 6 & $17.2(50)$ & $5.98(37.4)$ & 0.049 \\
& 12 & & $27.1(55.8)$ & $0(48.5)$ & 0.002 \\
Dexterity (COM) & 24 & & $34.2(57.6)$ & $2.04(56.7)$ & $<0.001$ \\
& & 6 & $17.4(19.4)$ & $0(18.6)$ & $<0.001$ \\
& 12 & & $23.6(32)$ & $3.13(31.1)$ & 0.004 \\
LUPUSQoL score & 24 & & $22.2(30.3)$ & $9.55(22.5)$ & 0.003 \\
(PH) & 12 & 6 & $9.5(49.9)$ & $-5.62(30.5)$ & 0.010 \\
& 24 & & $32.5(53.8)$ & $5.59(28.4)$ & $<0.001$ \\
& & $28.6(74.8)$ & $2.38(48.8)$ & 0.002 \\
\hline
\end{tabular}

All values are presented as median (interquartile range). DASH=Disabilities of the Arm, Shoulder, and Hand), $\mathrm{HAQ}=$ Health Assessment Questionnaire, LUPUS QoL=Lupus Quality of Life, $\mathrm{PH}=$ physical health domain, $\mathrm{DH}=$ dominant hand $\mathrm{JAW}=$ tripoid pinch, $\mathrm{COM}=$ combination.

Conclusion: Upper limb exercise, significantly improves hand function and quality of life in SLE patients.

Clinicaltrials.gov Id: NCT03802578

Disclosure of Interests: : None declared

DOI: 10.1136/annrheumdis-2019-eular.1411

\section{OP0249 \\ ANTIPHOSPHOLIPID SYNDROME (APS) IN SYSTEMIC LUPUS ERYTHEMATOSUS (SLE) LEADS TO A MORE SEVERE DISEASE}

Leyre Riancho-Zarrabeitia ${ }^{1}$, Victor Martinez Taboada ${ }^{2}$, Iñigo Rua-Figueroa ${ }^{3}$, Fernando Sánchez-Alonso ${ }^{4}$, María Galindo-Izquierdo ${ }^{5}$, Juan Ovalles ${ }^{6}$, Alejandro Olivé ${ }^{7}$, Antonio Fernandez-Nebro ${ }^{8}$, Jaime Calvo ${ }^{9}$, Raúl Menor Almagro ${ }^{10}$, Eva Tomero Muriel ${ }^{11}$, Esther Uriarte Isacelaya ${ }^{12}$, Alina Boteanu ${ }^{13}$, Mariano Andres ${ }^{14}$, Mercedes Freire González ${ }^{15}$, Gregorio Santos Soler ${ }^{16}$, Esther Ruiz Lucea ${ }^{17}$, Mónica Ibañez Barceló ${ }^{18}$, Ivan Castellví ${ }^{19}$, Carles Galisteo ${ }^{20}$, Víctor Quevedo Vila ${ }^{21}$, Enrique Raya ${ }^{22}, \mathrm{~J}_{\text {. Narváez }}{ }^{23}$, Lorena Expósito ${ }^{24}$, José A Hernandez Beriain25, Loreto Horcada ${ }^{26}$, Jose M PegoReigosa ${ }^{27} .{ }^{1} \mathrm{H}$ Sierrallana, Torrelavega, Spain; ${ }^{2} \mathrm{HU}$ Marqués Valdecilla, Santander Spain; ${ }^{3}$ H U Doctor Negrín, Las Palmas de Gran Canaria, Spain; ${ }^{4}$ Research Unit. SER, Madrid, Spain; ${ }^{5} \mathrm{HU} 12$ de Octubre, Madrid, Spain; ${ }^{6} \mathrm{H} U$ Gregorio Marañon, Madrid, Spain; ${ }^{7} \mathrm{HU}$ Germans Trias i Pujol, Badalona, Spain; ${ }^{8} \mathrm{HU}$ Carlos Haya, Malaga, Spain; ${ }^{9} \mathrm{HU}$ Araba, Alava, Spain; ${ }^{10}$ Hospital de Jerez, Jerez de la Frontera, Spain; ${ }^{11} \mathrm{HU}$ La princesa, Madrid, Spain; ${ }^{12} \mathrm{HU}$ Donosti, San Sebastian, Spain; ${ }^{13} \mathrm{HU}$ Ramón y Cajal, Madrid, Spain; ${ }^{14} \mathrm{HGU}$ Alicante, Alicante, Spain; ${ }^{15} \mathrm{HU}$ Juan Canalejo, La Coruña, Spain; ${ }^{16} \mathrm{H}$ Marina Baixa, Villajoyosa, Spain; ${ }^{17} \mathrm{HU}$ Basurto, Bilbao, Spain; ${ }^{18} \mathrm{H}$ Son Llatzer, Palma de Mallorca, Spain: ${ }^{19}$ Hospital de la Santa Creu i Sant Pau, Barcelona, Spain; ${ }^{20}$ HU Parc Tauli, Sabadell, Spain; ${ }^{21}$ Hospital Monforte, Monforte, Spain; ${ }^{22} \mathrm{HU}$ San Cecilio, Granada, Spain; ${ }^{23} \mathrm{HU}$ Bellvitge, L'Hospital de Llobregat, Spain; ${ }^{24}$ HU Canarias, Las Palmas de Gran Canaria, Spain; ${ }^{25} \mathrm{HU}$ Insular, Las Palmas de Gran Canaria, Spain; ${ }^{26} \mathrm{CHU}$ Navarra, Pamplona, Spain; ${ }^{27} \mathrm{CHU}$ Vigo, Vlgo, Spain

Background: Antiphospholipid antibodies (aPL) have been associated with organ damage and certain features in SLE patients.

Objectives: Our aim was to investigate the differences between SLE patients according to the presence of aPL and/or clinical antiphospholipid syndrome.

Methods: Patients from the RELESSER-T registry were included. RELESSER-T is a multicenter, hospital-based registry, with retrospective cross-sectional collection of data from a large representative sample of adult non-selected patients with SLE attending Spanish rheumatology services from the public national health system.

Results: We included 3651 SLE patients and 1368 were positive for aPL $(44.9 \%$ of patients were positive for anticardiolipin antibodies, $27.3 \%$ showed positivity for anti b2glycoprotein I and 24\% for lupus anticoagulant). Overall 2283 patients were classified as SLE no aPL, 528 as SLE-APS and 840 as SLE-aPL. Demographic data, clinical and laboratory features in the different groups are showed in Table 1. Regarding cardiovascular risk factors, SLE-APS patients had higher rates of hypertension, dyslipidemia and diabetes than SLE-aPL and SLE no aPL patients $(p<0.001, p<0.001$ and $p=0,022$, respectively). SLE-APS patients showed a lower prevalence of photosensitivity and higher frequencies of serositis, proteinuria ( $>0.5$ grs), urinary cell casts, seizures and psychosis $(p \leq 0.001)$. Over all, SLE-APS patients showed a lower rate of cutaneous manifestations and higher rates of neuropsychiatric, cardiac, pulmonary, renal, joint and ophthalmological manifestations (Table 1). In accordance with a more severe clinical profile, higher frequency of anti-DNA antibodies and hypocomplementemia were observed in the SLE-APS group $(p<0.001)$. In addition to a higher disease activity (SLEDAI), SLE APS patients presented more damage accrual with higher values in SLICC $(1.9+2.2$ in SLE APS, $0.9 \pm 1.4$ in SLE $\mathrm{APL}$ and $1.1 \pm 1.6, p<0.001)$ and Katz indexes $(3 \pm 1.8$ in SLE APS, $2.7 \pm 1.7$ in SLE aPL and 2.6 \pm 1.6 in SLE no aPL, $p<0.001)$. 


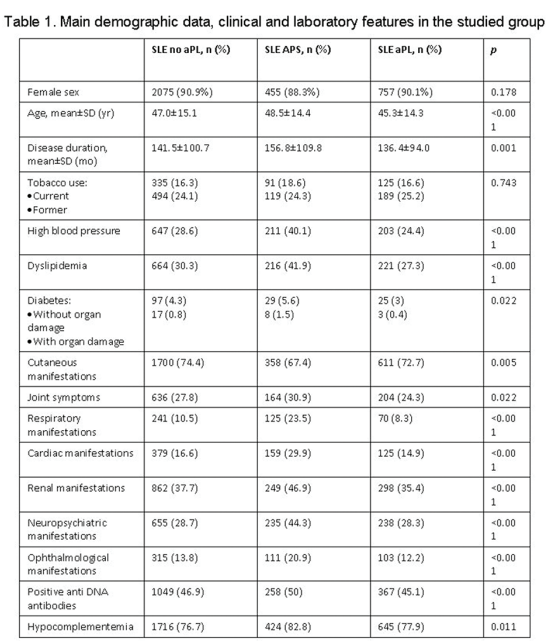

Conclusion: SLE-APS patients show a more severe clinical profile with higher frequency of major organ involvement and more damage accrual than SLE-aPL and SLE no APL.

Disclosure of Interests: : Leyre Riancho-Zarrabeitia Grant/research support from: Abbvie, Pfizer, UCB, MSD, GSK, Amgen, Roche travel grants, Victor Martinez Taboada: None declared, Iñigo Rua-Figueroa: None declared, Fernando Sánchez-Alonso: None declared, María Galindo-Izquierdo: None declared, Juan Ovalles: None declared, Alejandro Olivé Grant/research support from: ND, Consultant for: ND, Paid instructor for: ND, Speakers bureau: ND, Antonio FernandezNebro: None declared, Jaime Calvo Consultant for: Bristol-Myers Squibb, Janssen, Celgene, Sanofi Genzyme, Speakers bureau: Bristol-Myers Squibb, Raúl Menor Almagro: None declared, Eva Tomero Muriel: None declared, Esther Uriarte Isacelaya: None declared, Alina Boteanu: None declared, Mariano Andres: None declared, Mercedes Freire González: None declared, Gregorio Santos Soler: None declared, Esther Ruiz Lucea: None declared, Mónica Ibañez Barceló: None declared, Ivan Castellví Consultant for: I received fees less than 5000 USD as a consultant for Kern and Actelion, Paid instructor for: I received fees less than 2000USD as a instructor for Boehringer -Ingelheim, Novartis and Gebro, Speakers bureau: ND, Carles Galisteo: None declared, Víctor Quevedo Vila: None declared, Enrique Raya: None declared, J. Narváez Consultant for: BristolMyers Squibb, Lorena Expósito: None declared, José A Hernandez Beriain: None declared, Loreto Horcada: None declared, Jose M Pego-Reigosa: None declared DOI: 10.1136/annrheumdis-2019-eular.2475

\section{OP0250 \\ INFLUENTIAL FACTORS IN PROMOTING TREAT-TO- TARGET FOR SYSTEMIC LUPUS ERYTHEMATOSUS VIA EMPOWERING PATIENTS: A COHORT STUDY FROM CHINA BY SMART SYSTEM OF DISEASE MANAGEMENT (SSDM)}

Jianlin Huang ${ }^{1}$, Tong $\mathrm{Xie}^{2}$, Qiang Shu ${ }^{3}$, Jing Yang ${ }^{4}$, Yongfu Wang ${ }^{5}$, Hongzhi Wang ${ }^{6}$, Xiangyuan Liu' ${ }^{7}$, Cheng Zhao ${ }^{8}$, Xinwang Duan ${ }^{9}$, Guosheng Wang ${ }^{10}$, Rui Wu ${ }^{11}$, Yanjie Hao ${ }^{12}$, Yuhua Jia ${ }^{13}$, Fei Xiao ${ }^{13}$, Lingyun Sun ${ }^{14}$, SSDM Collaboration Group. ${ }^{1}$ The sixth Affiliated Hospital of Sun Yat-sen University, Guangzhou, China; ${ }^{2}$ Affiliated hospital of Guangdong medical University, Zhanjiang, China; ${ }^{3}$ Qilu Hospital of Shandong University, Jinan, China; ${ }^{4}$ Central Hospital of Mian Yang, Mian Yang, China; ${ }^{5}$ The First Affiliated Hospital of BaoTou Medical College, Bao Tou, China; ' JiaXing First Hospital, Jiaxing, China; ${ }^{7}$ Peking University Third Hospital, Beijing, China; ${ }^{8}$ The First Affiliated Hospital of Guangxi Medical University, Nanning; ${ }^{9}$ The Second Affiliated Hospital of Nanchang University, Nanchang, China; ${ }^{10}$ Anhui Provincial Hospital, Hefei, China; ${ }^{11}$ The First Affiliated Hospital of Nanchang University, Nanchang, China; ${ }^{12}$ Peking University First Hospital, Beijing, China; ${ }^{13}$ Shanghai Gothic Internet Technology Co., Ltd., Shanghai, China; ${ }^{14}$ The Affiliated Drum Tower Hospital of Nanjing University Medical School, Nanjing, China

Background: T2T is routine in RA, but no comparable standard has been defined for SLE. In 2015, the definition of Lupus Low Disease Activity State (LLDAS) was generated by Asia-Pacific Lupus Collaboration, and the preliminary validation demonstrated its attainment to be associated with improved outcomes in SLE. A SLEDAI-2K score lower than 4 is the main criteria for LLDAS. SSDM is an interactive mobile disease management application, including application systems for both the doctors and patients. The patients can perform self-assessment, including SLEDAI and medical records entry through the mobile application. The data is synchronized to the SSDM of authorized rheumatologists and stored in cloud database.
Objectives: To evaluate the patterns of T2T and related influential factors among SLE patients after applying SSDM in real world.

Methods: Patients were trained to master SSDM by rheumatologists in clinics. The first assessment for SLEDAI was performed as the baseline. Patients were required to perform repeated assessments after leaving the clinics.

Results: From July 2015 to Jan 2019, 1,090 SLE patients from 88 hospitals were followed up for more than 12 months through SSDM, and the results were summarized in Table 1. The ratio of T2T achievers was $52.84 \%(576 / 1,090)$ at the baseline and improved significantly to $68.35 \%(745 / 1,090)$ after a 12 -month follow-up, $p<0.01$. Among T2T achievers at the baseline, $77.08 \%$ (444/576) maintained T2T, and $22.92 \%(132 / 576)$ relapsed. Of patients who didn' $t$ achieve T2Tat baseline, 58.56\% (301/514) of the them achieve T2T after 12-month followup.

The impact of the times of self-assessment for SLEDAI on T2T has been analyzed. The more frequent of the self-assessments being conducted by patients, the higher improvement of T2T rate will be. We performed linear regression analysis of variables in statistics and parameter estimation by least square method. The improvement of T2T rate(y) was positively correlated with times of self-assessment for SLEDAI $(x)$ independently. The regression equation as " $y=0.0324 x+$ $0.0226 \mathrm{R} 2=0.7717 ", p<0.01$. (Figure 1 )

Conclusion: After proactive disease management via SSDM, the rate of T2T in SLE patients increased significantly. Patients with SLEDAI-2K $\leq 4$ score at baseline had a significantly higher retention rate. The patients who performed more self-assessments through SSDM had lower probability of relapse and higher rate of T2T maintaining and achievement. SSDM is a valuable tool for long term SLE follow-up through empowering patients.

Table1:

\begin{tabular}{lcccccc}
\hline $\begin{array}{l}\text { BaselinelLast } \\
\text { follow-up }\end{array}$ & Number & $\%$ & SLEDAI $\leq 4$ & $\%$ & SLEDAl $\geq 5$ & $\%$ \\
\hline SLEDAl $\leq 4$ & 576 & $52.84 \%$ & 444 & $77.08 \%$ & 132 & $22.92 \%$ \\
SLEDAI $\geq 5$ & 514 & $47.16 \%$ & 301 & $58.56 \%$ & 213 & $41.44 \%$ \\
Total & 1090 & $100 \%$ & 745 & $68.35 \%$ & 345 & $31.65 \%$ \\
\hline
\end{tabular}

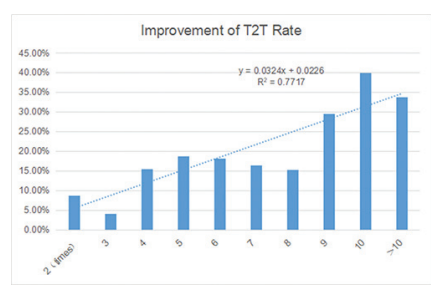

Figure 1

Disclosure of Interests: None declared

DOI: 10.1136/annrheumdis-2019-eular.5257

\section{OP0251 SLE PATIENTS FROM NORTH AMERICA ARE OLDER WITH LESS SEROLOGIC ACTIVITY THAN OTHER POPULATIONS IN INTERNATIONAL CLINICAL TRIALS}

Ewa Olech ${ }^{1}$, Eduard van Rijen ${ }^{1}$, Ali Ashrafzadeh ${ }^{1}$, Alexander Kant ${ }^{1}$, Joan T. Merrill'. ${ }^{1} I Q V I A$, Rheumatology Center of Excellence, Las Vegas, United States of America; ${ }^{2}$ Oklahoma Medical Research Foundation, Oklahoma City, United States of America

Background: Regional differences have been identified as potential confounders of SLE clinical trial results. Recently, no difference between treatment and placebo was observed in the US lupus patients when a significant treatment effect was observed in Europe ${ }^{1}$.

Objectives: To compare SLE serologic features/markers of active disease between different geographic regions in recent multinational clinical trials.

Methods: Laboratory data of 1005 subjects from four global randomized SLE clinical trials at baseline were examined. Mean/median C3 and C4 complement levels, prevalence of low $\mathrm{C} 3$ or $\mathrm{C} 4$ (Low C3/C4), positive anti-double stranded DNA (DNA), anti-Extractable Nuclear Antibodies (ENA), and high-titer Antinuclear Antibody (ANA $\geq 1: 640)$ in North America (NA) patients were compared to Asia (AS), Latin America (LA), Africa (AF), Western Europe (WE), and Eastern Europe (EE) Results: NA patients were significantly older than patients in LA, AF, or AS but not WE or EE. Not surprisingly, they also had higher complement levels and the lowest rates of low $\mathrm{C} 3 / \mathrm{C} 4$, DNA, ENA, and ANA $\geq 1: 640$. Our data confirm that age is an important factor in the prevalence of low complement and autoantibodies. However, there remained a marked difference in serologic activity between $\mathrm{NA}$ and $\mathrm{EE}$, despite being close in age. 